Article

\title{
Long-Term Incidence of Total Knee Arthroplasty after Open Reduction and Internal Fixation of Proximal Tibial and Distal Femoral Fractures: A Nationwide Cohort Study
}

\author{
Kuang-Ting Yeh ${ }^{1,2}{ }^{1}$, Wen-Tien $W u^{1,2,3}$, Ru-Ping Lee ${ }^{3}\left(\mathbb{C}\right.$, Chen-Chie Wang ${ }^{2,4}$, Jen-Hung Wang 5 (i) \\ and Ing-Ho Chen 1,2,* \\ 1 Department of Orthopedics, Hualien Tzu Chi Hospital, Buddhist Tzu Chi Medical Foundation, \\ Hualien 970473, Taiwan; micrograft@tzuchi.com.tw (K.-T.Y.); timwu@tzuchi.com.tw (W.-T.W.) \\ 2 School of Medicine, Tzu Chi University, Hualien 970374, Taiwan; xaiver-wang@yahoo.com.tw \\ 3 Institute of Medical Science, Tzu Chi University, Hualien 970374, Taiwan; fish@gms.tcu.edu.tw \\ 4 Department of Orthopedic Surgery, Taipei Tzu Chi Hospital, Buddhist Tzu Chi Medical Foundation, \\ New Taipei City 231405, Taiwan \\ 5 Department of Medical Research, Hualien Tzu Chi Hospital, Buddhist Tzu Chi Medical Foundation, \\ Hualien 970473, Taiwan; paulwang@tzuchi.com.tw \\ * Correspondence: ihchen@tzuchi.com.tw; Tel.: +886-3-8561825 (ext. 14713)
}

\section{check for} updates

Citation: Yeh, K.-T.; Wu, W.-T.; Lee, R.-P.; Wang, C.-C.; Wang, J.-H.; Chen, I.-H. Long-Term Incidence of Total Knee Arthroplasty after Open Reduction and Internal Fixation of Proximal Tibial and Distal Femoral Fractures: A Nationwide Cohort Study. J. Clin. Med. 2021, 10, 5685. https://doi.org/10.3390/jcm10235685

Academic Editors: Kevin L. Garvin and Rocco Papalia

Received: 8 November 2021

Accepted: 1 December 2021

Published: 2 December 2021

Publisher's Note: MDPI stays neutral with regard to jurisdictional claims in published maps and institutional affiliations.

Copyright: (c) 2021 by the authors. Licensee MDPI, Basel, Switzerland. This article is an open access article distributed under the terms and conditions of the Creative Commons Attribution (CC BY) license (https:// creativecommons.org/licenses/by/ $4.0 /)$.

\begin{abstract}
Knee fractures often require open reduction internal fixation (ORIF) for knee function recovery. More than one fifth of patients with knee fractures subsequently develop posttraumatic arthritis, and over $5 \%$ of them need total knee arthroplasty (TKA). We conducted this nationwide retrospective cohort study using the data of 2,000,000 people in the general population who received TKA and were followed up in the 17-year period 2001-2017, through random sampling of the Taiwan National Health Insurance Research Database. We matched the ORIF and non-fracture groups by a propensity score, based on age, sex, index date of surgery, and comorbidities enrolled in CCI calculated at a 1:1 ratio. The average proportion of subjects receiving TKA after ORIF for distal femoral or proximal tibial fractures was 2.0 per 1000 person-years, which was significantly higher than that in the non-fracture group. Patients aged 20-65 years and males represented a significantly higher proportion of subjects receiving TKA after ORIF than that in the non-fracture group. The proportion of subjects receiving TKA for the 20-65-year subgroup of the ORIF group was $4 \%$, and that for the male subgroup was $1.5 \%$; both rates increased over the 17-year follow-up period. More aggressive intraoperative and postoperative adjuvant therapies may be necessary for these subgroups.
\end{abstract}

Keywords: total knee arthroplasty; proximal tibial fracture; distal femoral fracture; nationwide-based cohort study; propensity score matching method

\section{Introduction}

Fractures of the distal femur, proximal tibia, and patella often engender inferior knee function [1], poor health-related quality of life [2], and further development of posttraumatic knee arthritis [3]. Posttraumatic knee arthritis occurs in approximately $23 \%$ to $36 \%$ of cases following intra-articular fractures [4]. This incidence does not appear to have decreased over time, despite advancements in fracture management and care strategies [5]. Moreover, posttraumatic knee arthritis is a critical risk factor for an increased rate of total knee arthroplasty (TKA), in addition to a relatively long human lifespan and an increased rate of obesity [6]. A systematic review suggested the optimal age for TKA was in the early 70s because patients within this age range could achieve the optimal passive range of motion after surgery [7]. Moreover, patients younger than 55 years were reported to have a significant effect on major aseptic revision rates [8]. A study also revealed that the rates of in-hospital adverse events after TKA decreased gradually from 2010 to 2017; however, critical risk factors for severe adverse events included the male sex [9]. Another study reported 
that the recurrence rate of postoperative flexion contracture was significantly higher in men than in women [10]. Therefore, age and sex differences, which are significantly correlated with future knee function and quality of life, appear to be critical factors for TKA-related knee function. Nevertheless, few large population cohort studies have focused on the influence of these factors on TKA rates after knee trauma. The use of conventional clinical approaches to investigate the relationship between open reduction internal fixation (ORIF) surgery for proximal tibial or distal femoral fractures and subsequent TKA is challenging because TKA is an infrequent complication after ORIF surgery. To fill the aforementioned research gap, we conducted this population-based retrospective study to evaluate the proportion of subjects receiving TKA and the age- and sex-related risk factors for TKA following ORIF surgery, for distal femoral or proximal tibial fractures.

\section{Materials and Methods}

Our study protocol was approved by the Research Ethics Committee of Hualien Tzu Chi Hospital (IRB108-242-C). We collected data from the Taiwan National Health Insurance Research Database (NHIRD), which is provided by the National Health Insurance Administration, Ministry of Health and Welfare, and managed by the National Health Research Institutes (NHRI). The NHIRD holds data on $99.7 \%$ of the population (nearly 23 million people) in Taiwan. Longitudinal Health Insurance Databases (LHID2000), which randomly sampled 2 million beneficiaries from the original NHIRD for the year 2000, was adopted for this study. The representativeness of LHIDs has been validated by NHRI. We included the data of patients who underwent ORIF surgery for distal femoral or proximal tibial fractures and subsequently received TKA between 1 January 2001 and 31 December 2017 (ORIF group). For the non-fracture group, we also collected the data for $2,000,124$ people in the general population through random sampling from the NHIRD (Figure 1). Relevant disease and surgical codes are listed in Table S1 (Supplementary Material). The comorbidities included in this study were based on those used in the Charlson Comorbidity Index (CCI) [11]. The case definition of each comorbidity was based on diagnostic codes, which have been employed and validated in other studies using claims databases. These comorbidities were identified by the presence of either at least two entries of diagnostic codes in the outpatient records, or one occurrence of discharge codes in hospitalization records, in the year before the index date.

We used the following methodology: A propensity score matching method was applied to reduce selection bias between the study groups. Age, sex, index year date of surgery, and comorbidities enrolled in CCI calculated at a 1:1 ratio were selected as independent variables. The Greedy method was used for matching, at a 1:1 ratio, the study groups, with a caliper width of 0.2 -fold the standard deviation of the propensity score between the study groups. The study outcome was subsequent to TKA performed after index surgery for distal femoral or proximal tibial factures. All available data were included in this study, and no additional unpublished data were included. The subgroup analysis was then performed by dividing all the patients into two groups by age (20-65 years and $>65$ years) and by sex (male and female). Interaction tests were employed to determine the subgroup effects of age and sex on the TKA risk.

Continuous variables are presented as means and standard deviations, and categorical variables are presented as number of cases and percentages. Continuous between-group variables were compared using Student's $t$-test, and categorical variables were assessed with a chi-square test. These tests were used to compare the characteristics of both groups. Data were evaluated using univariable and multivariable Cox regression analyses. Survival curves were estimated according to the Kaplan-Meier procedure, and groups were compared with use of the log-rank test. All statistical analyses were performed using SAS version 9.4 (SAS Institute Inc., Cary, NC, USA) and Stata 16.1 (StataCorp, College Station, TX, USA). We considered $p$-values of $<0.05$ as statistically significant. 


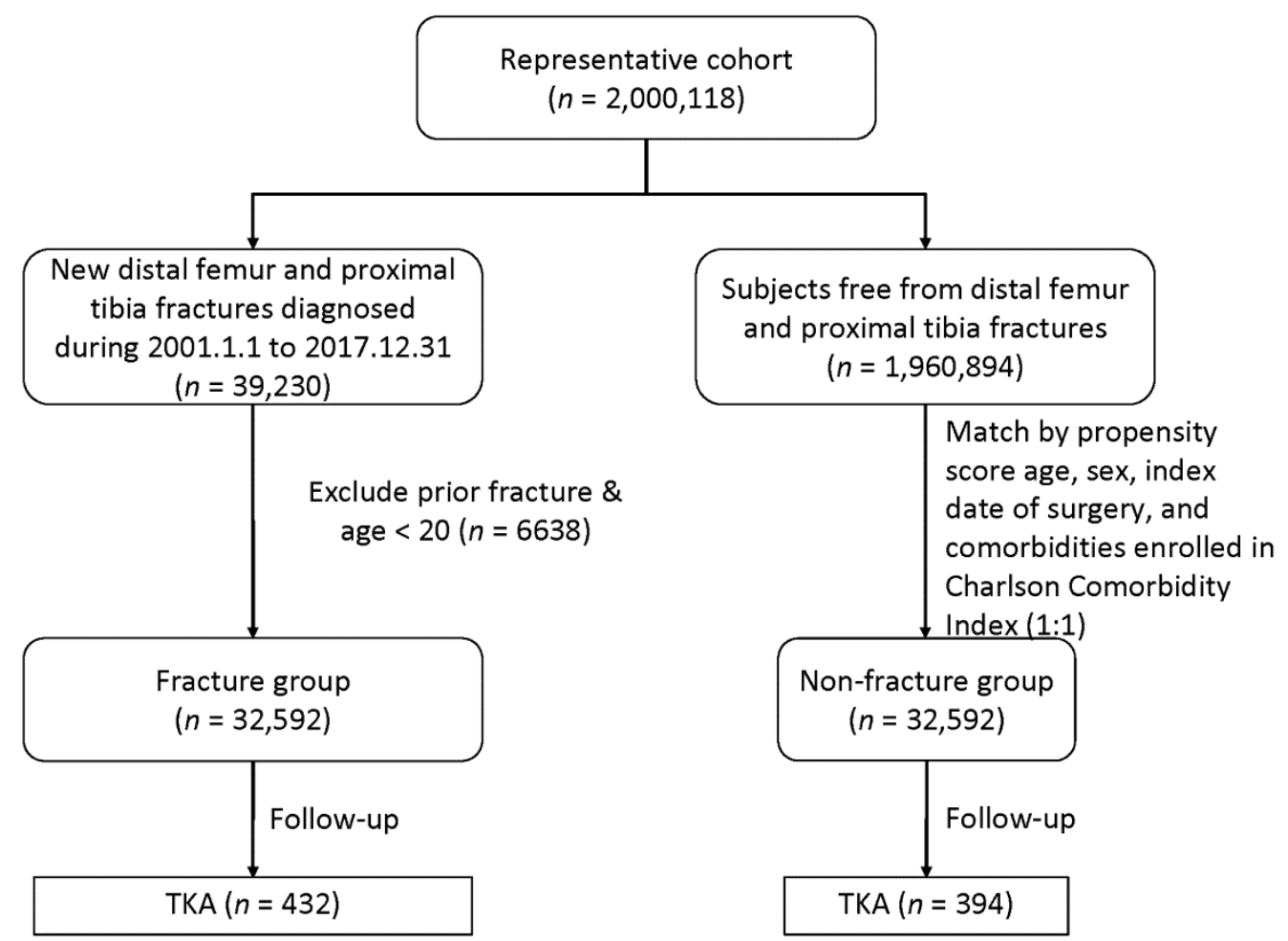

Figure 1. The study flow chart.

\section{Results}

The 1:1 propensity score matching yielded 32,592 patients in the ORIF group and 32,592 individuals in the non-fracture group (Table 1). The TKA rates in the ORIF and non-fracture groups were 2.0 and 1.6 per 1000 person-years, respectively (Table 2). From the Cox proportional hazard regression model, with adjustments for all baseline characteristics shown in Table 1, we observed that the adjusted hazard ratio (aHR) derived for the ORIF group relative to the non-fracture group was 1.23 (1.07-1.41; Table 2), which was determined to be significant ( $p=0.004$; Table 2$)$. The further subgroup analysis revealed that patients aged 20-65 years in the ORIF group had a significantly higher risk of TKA than their counterparts in the non-fracture group $(\mathrm{aHR}=1.94,1.51-2.48)$. Male patients in the ORIF group also had a significantly higher risk of TKA than their counterparts in the non-fracture group $(\mathrm{aHR}=1.56,1.19-2.04$; Table 3$)$. The effects of age and sex on the TKA risk were significant based on $p$ for interaction results (Table 3 ). The proportion of subjects receiving TKA in the 20-65-year subgroups of the ORIF and non-fracture groups increased linearly over time. A significantly higher risk of TKA was also observed in the ORIF group when compared with the non-fracture group (Figure 2A). The same distribution was also noted in the male subgroups of both the non-fracture and ORIF groups (Figure 2B). After 17 years, the proportion of subjects receiving TKA for the 20-65-year subgroup of the ORIF group was $4 \%$, and that for the male subgroup was $1.5 \%$ (Figure 2 ).

Table 1. Baseline characteristics and comorbidity.

\begin{tabular}{cccc}
\hline & \multicolumn{3}{c}{ Propensity Score Matching (1:1) } \\
\hline & $\begin{array}{c}\text { Non-Fracture } \\
(\boldsymbol{n = 3 2 , 5 9 2 )}\end{array}$ & $\begin{array}{c}\text { Fracture } \\
(\boldsymbol{n = 3 2 , 5 9 2 )}\end{array}$ & $p$-Value \\
\hline Age (y/o) & $58.75 \pm 20.01$ & $58.46 \pm 19.92$ & 0.058 \\
Age Group & & & 0.072 \\
$<65$ y/o & $17,985(55.2 \%)$ & $18,213(55.9 \%)$ & \\
$\geqq 65$ y/o & $14,607(44.8 \%)$ & $14,379(44.1 \%)$ & \\
\hline
\end{tabular}


Table 1. Cont.

\begin{tabular}{cccc}
\hline & \multicolumn{2}{c}{ Propensity Score Matching (1:1) } \\
\hline & $\begin{array}{c}\text { Non-Fracture } \\
(\boldsymbol{n}=\mathbf{3 2 , 5 9 2 )}\end{array}$ & $\begin{array}{c}\text { Fracture } \\
(\boldsymbol{n}=\mathbf{3 2 , 5 9 2 )}\end{array}$ & $p$-Value \\
\hline Male (\%) & $16,849(51.7 \%)$ & $16,873(51.8 \%)$ & 0.851 \\
Comorbidity & & & \\
HTN & $10,134(31.1 \%)$ & $10,029(30.8 \%)$ & 0.374 \\
DM & $5803(17.8 \%)$ & $5747(17.6 \%)$ & 0.566 \\
Hyperlipidemia & $3377(10.4 \%)$ & $3440(10.6 \%)$ & 0.420 \\
Chronic renal failure & $936(2.9 \%)$ & $1015(3.1 \%)$ & 0.069 \\
CAD & $2835(8.7 \%)$ & $2879(8.8 \%)$ & 0.542 \\
CVA & $3183(9.8 \%)$ & $3128(9.6 \%)$ & 0.466 \\
Alcohol-induced mental disorders & $50(0.2 \%)$ & $70(0.2 \%)$ & 0.068 \\
Alcohol dependence syndrome & $84(0.3 \%)$ & $112(0.3 \%)$ & $0.045 *$ \\
Drug dependence & $37(0.1 \%)$ & $44(0.1 \%)$ & 0.436 \\
Chronic liver disease & $1636(5.0 \%)$ & $1697(5.2 \%)$ & 0.278 \\
Iron deficiency anemia & $310(1.0 \%)$ & $384(1.2 \%)$ & $0.005 *$ \\
Depression & $1432(4.4 \%)$ & $1402(4.3 \%)$ & 0.564 \\
Dementia & $1233(3.8 \%)$ & $1204(3.7 \%)$ & 0.549 \\
Peripheral vascular disease & $330(1.0 \%)$ & $385(1.2 \%)$ & $0.039 *$ \\
\hline
\end{tabular}

y/o: years old. Data are presented as $n$ and percentage. ${ }^{*}$ A value of $p<0.05$ was considered statistically significant after test.

Table 2. Risk of TKA in patients with and without fracture.

\begin{tabular}{|c|c|c|}
\hline & \multicolumn{2}{|c|}{ Propensity Score Matching (1:1) } \\
\hline & \multicolumn{2}{|c|}{ Fracture } \\
\hline & Yes & No \\
\hline Patient numbers & 32,592 & 32,592 \\
\hline TKA cases & 432 & 394 \\
\hline Person-years & 219,264 & 244,653 \\
\hline Incidence rate ${ }^{a}$ & 2.0 & 1.6 \\
\hline $\begin{array}{l}\text { Univariable model } \\
\text { crude HR }(95 \% \mathrm{CI}) \\
p \text {-value }\end{array}$ & $\begin{array}{c}1.16(1.01-1.33) \\
0.035 *\end{array}$ & 1 (ref.) \\
\hline $\begin{array}{c}\text { Multivariable model } \mathrm{b} \\
\text { aHR }(95 \% \mathrm{CI}) \\
p \text {-value }\end{array}$ & $\begin{array}{c}1.23(1.07-1.41) \\
0.004 *\end{array}$ & 1 (ref.) \\
\hline
\end{tabular}

aHR: adjusted hazard ratio; CI: confidence interval; HR: hazard ratio; ref: reference. ${ }^{\text {a }}$ Per 1000 person-years

${ }^{b}$ Multivariable Cox proportional hazard regression model with adjustment for all baseline characteristics shown in Table $1 .{ }^{*}$ A value of $p<0.05$ was considered statistically significant after test.

Table 3. Subgroup analysis of Cox's regression model for the association between fracture and TKA.

\begin{tabular}{|c|c|c|c|c|c|}
\hline \multirow{2}{*}{ Variables } & \multicolumn{5}{|c|}{ Propensity Score Matching (1:1) } \\
\hline & Crude HR ${ }^{a}(95 \%$ CI) & $p$-Value & Adjusted HR a $(95 \%$ CI) & $p$-Value & $p$ for Interaction \\
\hline \multicolumn{6}{|l|}{ Main model } \\
\hline Non-fracture & 1.00 & & 1.00 & & \\
\hline Fracture & $1.16(1.01-1.33)$ & $0.035 *$ & $1.23(1.07-1.41)$ & $0.004 *$ & \\
\hline \multicolumn{6}{|l|}{ Age } \\
\hline \multicolumn{6}{|l|}{$20-65$ y/o } \\
\hline Non-fracture & 1.00 & & 1.00 & & \multirow{5}{*}{$<0.001 *$} \\
\hline Fracture & $1.94(1.52-2.49)$ & $<0.001 *$ & $1.94(1.51-2.48)$ & $<0.001 *$ & \\
\hline$\geqq 65 \mathrm{y} / \mathrm{o}$ & & & & & \\
\hline Non-fracture & 1.00 & & 1.00 & & \\
\hline Fracture & $0.97(0.82-1.15)$ & 0.746 & $0.96(0.81-1.14)$ & 0.634 & \\
\hline
\end{tabular}


Table 3. Cont.

\begin{tabular}{|c|c|c|c|c|c|}
\hline \multirow{2}{*}{ Variables } & \multicolumn{5}{|c|}{ Propensity Score Matching (1:1) } \\
\hline & Crude $\mathrm{HR}^{\text {a }}(95 \% \mathrm{CI})$ & $p$-Value & Adjusted HR a $(95 \%$ CI $)$ & $p$-Value & $p$ for Interaction \\
\hline \multicolumn{6}{|l|}{ Gender } \\
\hline \multicolumn{6}{|l|}{ Male } \\
\hline Non-fracture & 1.00 & & 1.00 & & \multirow{5}{*}{0.075} \\
\hline Fracture & $1.39(1.07-1.82)$ & $0.015 *$ & $1.56(1.19-2.04)$ & $0.001 *$ & \\
\hline Female & & & & & \\
\hline Non-fracture & 1.00 & & 1.00 & & \\
\hline Fracture & $1.08(0.92-1.27)$ & 0.368 & $1.13(0.96-1.33)$ & 0.131 & \\
\hline
\end{tabular}

CI, confidence interval; HR, hazard ratio. ${ }^{a}$ Cox's proportional hazards model. * A value of $p<0.05$ was considered statistically significant after test.
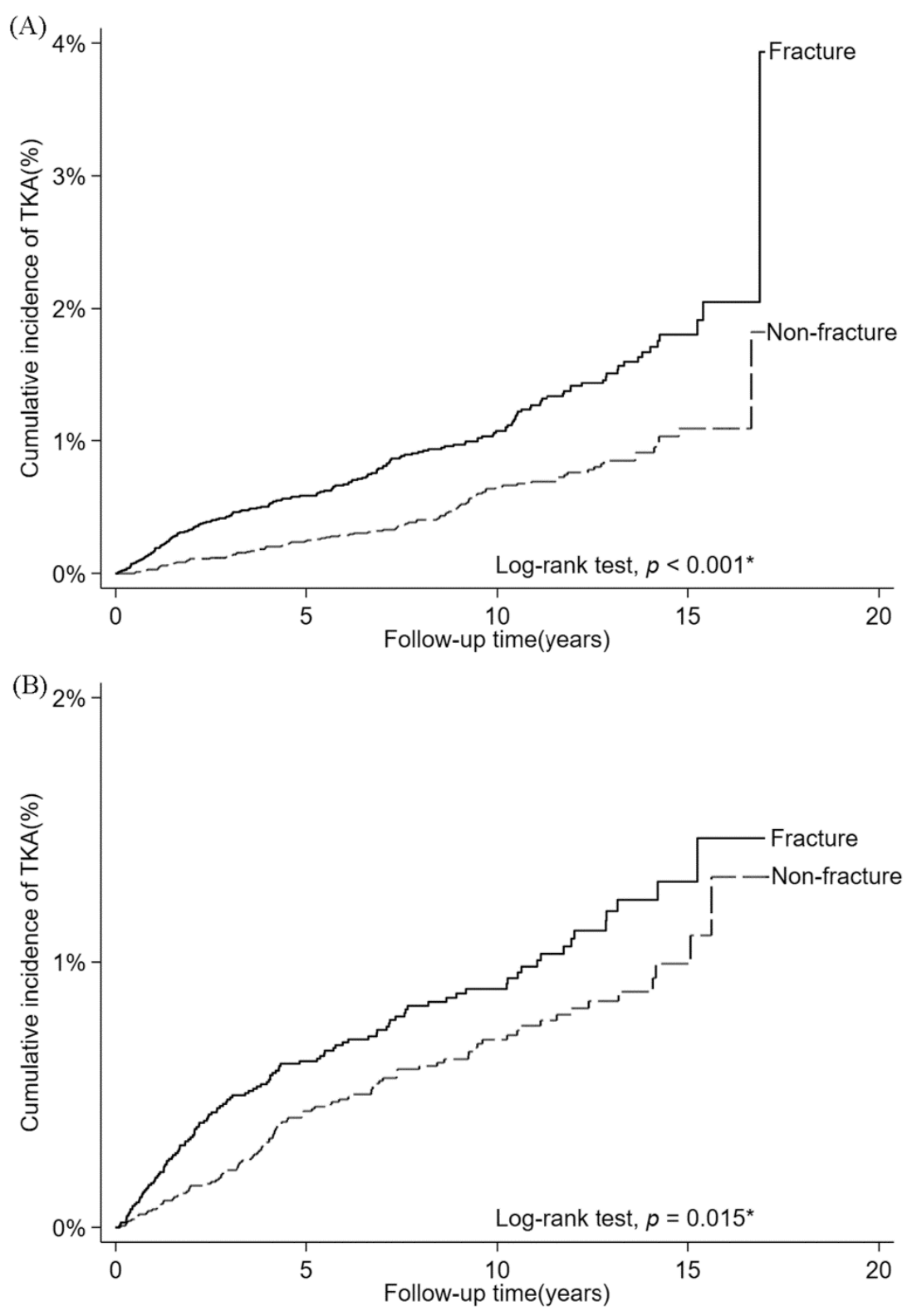

Figure 2. The proportion of subjects receiving TKA of the fracture group and the non-fracture group. (A) The 20-65-year subgroups of the ORIF and non-fracture groups. (B) The male subgroups of the ORIF and non-fracture groups. * A value of $p<0.05$ was considered statistically significant after test. 


\section{Discussion}

Distal femoral and proximal tibial fractures often include intra-articular injury and further lead to posttraumatic osteoarthritis. Most cases of degenerative arthritis of the knee in patients younger than 50 years are due to posttraumatic osteoarthritis [12]. Reports of the management of geriatric knee fractures have increased in recent decades because people now have a longer lifespan. TKA should be performed on patients with advanced knee arthritis and unacceptable knee function. According to patient-reported outcomes from a national cross-sectional study, female patients aged older than 40 years had high odds ratios for poor functional outcomes in long-term follow-up after a knee fracture [13]. A previous study reported that a major factor for poor functional status after trauma was posttraumatic osteoarthritis, the incidence of which was as high as 75\% [14]. Moreover, a 2014 Canadian nationwide matched population-based cohort study reported that the overall incidence of tibial plateau fractures leading to end-stage posttraumatic osteoarthritis that required further TKA was $7.3 \%$ at 10 years after ORIF; relevant risk factors included increasing age, bicondylar fracture, concomitant meniscal tear injury, increased medical comorbidities, and the female sex [15]. A 20-year Danish nationwide cohort study revealed that patients with knee fractures had a 1.6 times greater risk of TKA at 3 years after trauma and throughout their lifetimes compared to other individuals; the study indicated that surgical treatment of knee fractures, or external fixation, remained the major relative risk factor [16]. In our study, the TKA rate after ORIF for proximal tibial and distal femoral fractures was 2.0 per 1000 person-years, and the 17-year incident rate of TKA was less than $4 \%$, which are comparable to those reported in the literature [15-17]. This indicates that, although severe knee fractures that require ORIF may be correlated with a high risk of TKA, adequate ORIF for knee fractures is still a crucial procedure for preserving knee function after traumatic fractures.

The principle of ORIF for intra-articular knee fractures includes anatomical articular surface restoration, condylar width restoration, mechanical axis alignment restoration, joint stability reconstruction, and soft tissue repair [18]. Currently, strategies extensively applied for treating or managing distal femoral and proximal tibial fractures involve locked plating of fractures and minimally invasive approaches, such as anatomical reduction of fractures, which can considerably reduce the development of advanced posttraumatic knee osteoarthritis and facilitate postoperative functional recovery [19-21]. This thus explains the lower TKA rates in our cohort study compared with those in the literature. In our study, the TKA rates observed for male patients and younger patients were nearly two times higher than those observed for the non-fracture group. The possible reasons for this finding are multifactorial, including working status, early return to work because of economic factors, or poor compliance with physical therapy. Such patients may require more aggressive postoperative management approaches and follow-up protocols, including early detection of the progression of posttraumatic arthritis with serum biomarker levels [22], radiographic studies [23], prompt intervention with anti-inflammatory and regeneration therapy [24], aggressive rehabilitation programs for muscle strength around the knee joint, and adequate protective braces suitable for early return to work after ORIF.

We originally matched the fracture and non-fracture groups through a 1:2 ratio by age, sex, and index date, and the analytical results of this matching method are represented as Figure S1 and Tables S2-S4. To further eliminate significant differences in the comorbidities between the groups, we then matched the ORIF and non-fracture groups by propensity score, age, sex, index date of surgery, and comorbidities enrolled in CCI calculated at a 1:1 ratio. The results of both methods were similar, and this indicates the validity of our results.

A strength of this study is the large sample size; the comprehensive coverage of the National Health Insurance system (covering > 95\% of the Taiwanese population) may have minimized selection and nonresponse biases. However, this study has some limitations. First, the data were acquired from NHIRD, which registers only surgical fee codes, and the severity or classification of the fractures could not be determined for further correlation 
with the rate of future TKA incidence. Second, the data on lifestyle factors, personal characteristics, and detailed postoperative adjuvant treatment, which may be influential sources of bias, were unavailable because the NHIRD does not provide this information. Third, because our results are based on data from Taiwan, the present findings may not be directly generalizable to Caucasian or African populations.

\section{Conclusions}

The average proportion of subjects receiving TKA after ORIF for distal femoral or proximal tibial fractures was 2.0 per 1000 person-years and was significantly higher than that in the non-fracture group, especially in the subgroups comprising individuals aged 20-65 years and men. More aggressive intraoperative and postoperative adjuvant therapies and protocols for these subgroups may be necessary. The proportion of subjects receiving TKA after knee fracture increased gradually during the 17-year follow-up period. The long-term proportion of subjects receiving TKA in our study is lower than that in the literature, and this difference is attributable to race differences or recent surgical advances.

Supplementary Materials: The following are available online at https:/ /www.mdpi.com/article/10 $.3390 /$ jcm10235685/s1, Table S1: ICD9 and ICD10 diagnosis codes. Table S2: Baseline characteristics and comorbidity according to age, sex, and index date at a 1:2 ratio. Table S3: Risk of TKA in patients with and without fracture according to age, sex, and index date at a 1:2 ratio. Table S4: Subgroup analysis of Cox's regression model for the association between fracture and TKA according to age, sex, and index date at a 1:2 ratio. Figure S1: The proportion of subjects receiving TKA of the fracture group and the non-fracture group according to age, sex, and index date at a 1:2 ratio. (A) The 20-65-year subgroups of the ORIF and non-fracture groups. (B) The male subgroups of the ORIF and non-fracture groups.

Author Contributions: Conceptualization, validation, and formal analysis, I.-H.C. and K.-T.Y.; methodology and software, J.-H.W.; investigation, K.-T.Y. and C.-C.W.; resources, J.-H.W.; data curation, W.-T.W., R.-P.L. and I.-H.C.; writing-original draft preparation, K.-T.Y.; writing-review and editing, W.-T.W., R.-P.L. and I.-H.C.; visualization, I.-H.C.; supervision, W.-T.W., R.-P.L. and I.-H.C.; project administration, W.-T.W.; funding acquisition, I.-H.C. All authors have read and agreed to the published version of the manuscript.

Funding: This research was funded by Tzu Chi Medical Mission Project 106-05-01, Buddhist Tzu Chi Medical Foundation (TCMMP 106-05-01).

Institutional Review Board Statement: This study was conducted according to the guidelines of the Declaration of Helsinki and approved by the Research Ethics Committee of Hualien Tzu Chi Hospital, Buddhist Tzu Chi Medical Foundation (IRB108-207-C and date of approval: 11 October 2019).

Informed Consent Statement: Patient consent was waived due to the National Health Insurance dataset consisting of de-identified secondary data used for research purposes, and the Research Ethics Committee of Hualien Tzu Chi Hospital gave a formal written waiver of the need for consent.

Data Availability Statement: Our data are third-party data, which are now managed by the Ministry of Health and Welfare. Others can access these datasets by application and following approval by our government. Our data are restricted by National Health Insurance, Department of Health of Taiwan. The government made the rule that all data be returned after this study finished. The data cannot be studied without approval. If there are further requests for the data, one should apply for the data from our National Health Insurance. The rules of how to apply for and use these data can be found at https: / / dep.mohw.gov.tw/DOS/mp-113.html. The authors confirm they had no special privileges or access to the data and that all interested, qualified researchers may access the data using the application process described.

Conflicts of Interest: The authors declare no conflict of interest. The funding sources had no influence on the study design or interpretation. 


\section{References}

1. Canadian Orthopaedic Trauma Society. Open reduction and internal fixation compared with circular fixator application for bicondylar tibial plateau fractures. Results of a multicenter, prospective, randomized clinical trial. J. Bone Joint Surg. Am. 2006, 88, 2613-2623. [CrossRef]

2. Sluys, K.P.; Shults, J.; Richmond, T.S. Health related quality of life and return to work after minor extremity injuries: A longitudinal study comparing upper versus lower extremity injuries. Injury 2016, 47, 824-831. [CrossRef]

3. LeBrun, C.T.; Langford, J.R.; Sagi, H.C. Functional Outcomes After Operatively Treated Patella Fractures. J. Orthop. Trauma 2012, 26, 422-426. [CrossRef] [PubMed]

4. $\quad$ Rademakers, M.V.; Kerkhoffs, G.M.M.J.; Sierevelt, I.N.; Raaymakers, E.L.F.B.; Marti, R.K. Operative Treatment of 109 Tibial Plateau Fractures: Five- to 27-Year Follow-up Results. J. Orthop. Trauma 2007, 21, 5-10. [CrossRef]

5. Aurich, M.; Koenig, V.; Hofmann, G. Comminuted intraarticular fractures of the tibial plateau lead to posttraumatic osteo-arthritis of the knee: Current treatment review. Asian J. Surg. 2018, 41, 99-105. [CrossRef]

6. Losina, E.; Thornhill, T.S.; Rome, B.N.; Wright, J.; Katz, J.N. The Dramatic Increase in Total Knee Replacement Utilization Rates in the United States Cannot Be Fully Explained by Growth in Population Size and the Obesity Epidemic. J. Bone Jt. Surg. Am. Vol. 2012, 94, 201-207. [CrossRef] [PubMed]

7. Lee, S.H.; Kim, D.H.; Lee, Y.S. Is there an optimal age for total knee arthroplasty?: A systematic review. Knee Surg. Relat. Res. 2020, 32, 1-11. [CrossRef]

8. Jorgensen, N.B.; McAuliffe, M.; Orschulok, T.; Lorimer, M.F.; de Steiger, R. Major aseptic revision following total knee replacement: A study of 478,081 total knee replacements from the Australian Orthopaedic Association National Joint Re-placement Registry. J. Bone Joint Surg. Am. 2019, 101, 302-310. [CrossRef] [PubMed]

9. Halawi, M.J.; Gronbeck, C.; Metersky, M.L.; Wang, Y.; Eckenrode, S.; Mathew, J.; Suter, L.G.; Eldridge, N. Time Trends in Patient Characteristics and In-Hospital Adverse Events for Primary Total Knee Arthroplasty in the United States: $2010-2017$. Arthroplast. Today 2021, 11, 157-162. [CrossRef]

10. Kinoshita, T.; Hino, K.; Kutsuna, T.; Watamori, K.; Tsuda, T.; Miura, H. Gender-specific difference in the recurrence of flexion contracture after total knee arthroplasty. J. Exp. Orthop. 2021, 8, 1-9. [CrossRef] [PubMed]

11. Vestergaard, V.; Pedersen, A.B.; Tengberg, P.T.; Troelsen, A.; Schrøder, H.M. 20-year trends of distal femoral, patellar, and proximal tibial fractures: A Danish nationwide cohort study of 60,823 patients. Acta Orthop. 2019, 91, 109-114. [CrossRef] [PubMed]

12. Honkonen, S.E. Degenerative Arthritis After Tibial Plateau Fractures. J. Orthop. Trauma 1995, 9, 273-277. [CrossRef]

13. Vestergaard, V.; Schrøder, H.M.; Hare, K.B.; Toquer, P.; Troelsen, A.; Pedersen, A.B. Patient-reported outcomes of 7133 distal femoral, patellar, and proximal tibial fracture patients: A national cross-sectional study with one-, three-, and five-year follow-up. Knee 2020, 27, 1310-1324. [CrossRef]

14. Schenker, M.L.; Mauck, R.L.; Ahn, J.; Mehta, S. Pathogenesis and prevention of posttraumatic osteoarthritis after in-tra-articular fracture. J. Am. Acad. Orthop. Surg. 2014, 22, 20-28. [CrossRef]

15. Wasserstein, D.; Henry, P.; Paterson, J.M.; Kreder, H.J.; Jenkinson, R. Risk of total knee arthroplasty after operatively treated tibial plateau fracture: A matched-population- based cohort study. J. Bone Joint Surg. Am. 2014, 96, 144-150. [CrossRef]

16. Vestergaard, V.; Pedersen, A.B.; Hare, K.B.; Schrøder, H.M.; Troelsen, A. Knee Fracture Increases TKA Risk After Initial Fracture Treatment and Throughout Life. Clin. Orthop. Relat. Res. 2020, 478, 2036-2044. [CrossRef]

17. Stake, S.N.; Gu, A.; Fassihi, S.C.; Ramamurti, P.; Bovonratwet, P.; Thakkar, S.C.; Golladay, G.J. Increased Revisions in Conversion Total Knee Arthroplasty After Periarticular Open Reduction Internal Fixation Compared With Primary Total Knee Arthroplasty: A Matched Cohort Analysis. J. Arthroplast. 2021, 36, 3432-3436.e1. [CrossRef]

18. Davis, J.T.; Rudloff, M.I. Posttraumatic Arthritis After Intra-Articular Distal Femur and Proximal Tibia Fractures. Orthop. Clin. N. Am. 2019, 50, 445-459. [CrossRef] [PubMed]

19. Hoffmann, M.F.; Jones, C.B.; Sietsema, D.L.; Tornetta, P., 3rd; Koenig, S.J. Clinical outcomes of locked plating of distal femoral fractures in a retrospective cohort. J. Orthop. Surg. Res. 2013, 8, 43. [CrossRef] [PubMed]

20. Harvin, W.H.; Oladeji, L.O.; Della Rocca, G.J.; Murtha, Y.M.; Volgas, D.A.; Stannard, J.P.; Crist, B.D. Working length and proximal screw constructs in plate osteosynthesis of distal femur fractures. Injury 2017, 48, 2597-2601. [CrossRef]

21. Kumar, V.; Singhroha, M.; Arora, K.; Sahu, A.; Beniwal, R.; Kundu, A. A clinico-radiological study of bicondylar tibial plateau fractures managed with dual locking plates. J. Clin. Orthop. Trauma 2021, 21, 101563. [CrossRef] [PubMed]

22. Khella, C.M.; Asgarian, R.; Horvath, J.M.; Rolauffs, B.; Hart, M.L. An Evidence-Based Systematic Review of Human Knee Post-Traumatic Osteoarthritis (PTOA): Timeline of Clinical Presentation and Disease Markers, Comparison of Knee Joint PTOA Models and Early Disease Implications. Int. J. Mol. Sci. 2021, 22, 1996. [CrossRef] [PubMed]

23. Mason, D.; Englund, M.; Watt, F.E. Prevention of posttraumatic osteoarthritis at the time of injury: Where are we now, and where are we going? J. Orthop. Res. 2021, 39, 1152-1163. [CrossRef] [PubMed]

24. Khella, C.; Horvath, J.; Asgarian, R.; Rolauffs, B.; Hart, M. Anti-Inflammatory Therapeutic Approaches to Prevent or Delay Post-Traumatic Osteoarthritis (PTOA) of the Knee Joint with a Focus on Sustained Delivery Approaches. Int. J. Mol. Sci. 2021, 22, 8005. [CrossRef] [PubMed] 